\title{
Calidad en la educación universitaria, desde el programa de ingeniería de sistemas: una visión cualitativa de la educación superior
}

\author{
René Ramírez-Fernández ${ }^{1}$ \\ Jhorquis Machado-Licona ${ }^{2}$ \\ Oscar Eduardo Fernández-Ramírez ${ }^{3}$
}

\begin{abstract}
Resumen
El presente documento divulga los resultados de la investigación "calidad en la educación universitaria, percepción de los estudiantes de la facultad de ingeniería" realizada en el programa de ingeniería de sistemas de una Universidad de la Ciudad de Cartagena; el objetivo de dicha investigación es analizar como los estudiantes del programa de ingeniería de sistemas observan la calidad de educación impartida en la Universidad; para el estudio se seleccionaron cuatro variables, currículo, formación docente, soporte administrativo e infraestructura, las cuales fueron evaluadas por los alumnos de segundo a decimo semestre; el método implementado es deductivo-inductivo y el enfoque es cualitativo. Dentro de los principales resultados, se pudo observar que las unidades de análisis, actualidad del currículo, tutorías y refuerzos, adecuación de las aulas y espacios de socialización, son lo que demandan mayor trabajo por parte de la Universidad.
\end{abstract}

\section{Palabras Claves}

Calidad, educación universitaria, currículo, infraestructura, academia

\begin{abstract}
This document disseminates the results of the research "quality in university education, perception of students of the faculty of engineering" carried out in the system engineering program of a University of the City of Cartagena; the objective of this research is to analyze how the students of the systems engineering program observe the quality of education taught at the University; four variables, curriculum, teacher training, administrative support and infrastructure were selected for the study, which were evaluated by students from second to tenth semester; the implemented method is deductive-inductive and the approach is qualitative. Among the main results, it was observed that the units of analysis, currentcurriculum, tutoring and reinforcements, adequacy of the classrooms and socialization spaces, are what demand the most work by the University.
\end{abstract}

\section{Keywords}

Quality, university education, curriculum, infrastructure, academy

Fecha de recepción: 11 de Junio de 2019, Fecha de evaluación: 17 de Julio de 2019, Fecha de aceptación: 14 de septiembre de 2019

\footnotetext{
1. Especialista en inteligencia artificial y sistemas expertos, Magister en educación. Director de autoevaluación en la corporación Universitaria Americana. Correo electrónico:ciber.ramirez@gmail.com. ORCID ID: https://orcid.org/0000-0001-9452-7580 2. Administrador de empresas de la Universidad Libre. Integrante del grupo de investigación Ciencia Libre, Correo electrónico: jhorquis_machado@unilibrectg.edu.co. ORCID ID: https://orcid.org/0000-0002-6987-7658

3. Administrador de empresas, Especialista en Alta Gerencia, Capitan de la Infanteria de Marina, Director de investigaciones de la escuela de formación de Infantería de Marina Correo electrónico: oscar.fernandez@armada.mil.co. ORCID ID: https://orcid.org/ 0000-0002-8673-5483
} 


\section{Introducción}

El papel que desempeña la Universidad dentro de la sociedad es relevante ya que esta es garante de la formación del capital humano que hará frente a la demanda laboral futura en los distintos ámbitos; formar profesionales con visión, con capacidad crítica y capaces de afrontar diversos roles desde lo profesional hasta lo investigativo; bajo esta perspectiva es necesaria la puesta en marcha de estrategias que permitan hacer frente a las necesidades cada vez más complejas del mercado laboral, por lo cual es importante no solo la actualización de los currículos sino, a través de los grupos de interés realizar una constante evaluación de la calidad de la educación impartida por las Universidades, desde factores de soporte como factores puramente académicos e investigativos.

Para autores como Camacaro (2010), el evaluar el concepto de calidad en la educación Universitaria es un proceso complejo que puede ser visto de distintas variables que se complementan entre sí para dar un acercamiento más exacto a lo que significa la calidad; la complejidad mencionada por el autor se puede observar desde la multidimensionalidad de los factores, partiendo de la premisa básica que las Universidades son organizaciones, con una visión, una proyección y una afectación más amplia sobre la sociedad. Algunos de las variables claves expuesta por Camacaro (2010) son:

\section{- La calificación y formación del profe-} sorado: La capacidad de los docentes, las habilidades, competencias, este esta se vincula con el nivel y tipo de formación del profesorado e inclusive con la experiencia adquirida en el desempeño habitual de sus actividades profesionales tanto dentro como fuera de la academia.
- La programación docente: se relaciona con un indicador que permite establecer un coeficiente que mide la relación número docentes frente al número de estudiantes; lo óptimo es que exista la cantidad de docentes necesarios para cubrir todo el estudiantado, a menor número docente $\mathrm{y}$ mayor número de estudiante es menor la posibilidad de que todos los estudiantes aprendan por igual.

\section{- Tecnología de la Información y las comu-} nicaciones: La Universidades deben contar con lo medio tecnológicos, modernos que permitan estar a la altura de los estudiantes del presente y del futuro, contando así con los canales tecnológicos que faciliten la interacción del docente tanto dentro como fuera de las aulas de clase, se habla de plataforma virtuales como Moodle, con equipos de cómputo modernos, con base de datos $\mathrm{y}$ repositorios entre otras muchas herramientas tecnológicas.

\section{- Estructura de la Organización Educati-}

va: Poseer una estructura organizativa que facilite la participación de los estudiantes en la construcción de una Universidad más acorde con las necesidades la sociedad, con una universidad moderna y equitativa, debe ser un objetivo estratégico de toda institución universitaria.

- Calidad en el Currículo: La estructura y la coherencia en la malla curricular, ofreciendo de este modo contenidos actualizados, con un cuerpo bien definido, manteniendo la paridad entre el método de enseñanza y el método de evaluación, además de la referenciación de materiales adecuados para la mejor comprensión de los diversos tópicos a tratar.

- Calidad de la enseñanza: El clima óptimo para el aprendizaje es uno de los factores que los grupos de interés deben garantizar, 
ya que en la medida que exista un clima propicio será mejor la aprehensión de conocimientos por parte del estudiantado, cabe aclara que el clima nos solo se ciñe a las aulas de clase sino a todas las estancias de la Universidad; del mismo modo los refuerzos, el seguimiento y control también hacen parte de la calidad de la enseñanza, aporta mucho al proceso de aprendizaje las base para evaluar y apoyar al proceso de afianzar conocimientos.

La calidad en la educación Universitaria debe cimentarse en los procesos de mejoramiento continuo, con lo cual se garantiza una educación actualizada y congruente con la realidad social.

\section{Calidad y calidad académica}

El componente multidimensional sumado a las distintas perspectivas de los grupos de interés convierte en un verdadero reto el definir con exactitud el concepto de calidad, sin obviar ningún matiz propio del término, para tener un mejor acercamiento a la compresión de calidad es necesario abordar las distintas propuestas conceptuales de autores modernos.

Definir calidad es confuso en gran parte debido a que las personas observan la calidad basado diferentes criterios, propios de las actividades que realizan dentro de las organizaciones; otra razón que hace complejo el definir calidad se debe a que el concepto evoluciona en la medida en que madura la profesión sobre la cual se aplica; la calidad se puede asumir como una función medible, basado en un atributo especifico aumentado; de otra parte desde la perspectiva del usuario el concepto de calidad entraña subjetividad al basarse en la expectativa que el subjeto tiene sobre un servicio o producto. (Evans et al., 2000)
La Real Academia de la Lengua la calidad se define en su principal acepción "la calidad es las propiedad o característica de un objeto, que facilita el evaluar su real valor". (RAE, 2012)

Para Ishikawa (1988) la calidad esta enlazada con el diseño, desarrollo o manufactura de productos o servicios guardando que sea satisfactorio para los clientes, por su utilidad y precio competitivo, esta definición toma a consideración el cliente como elemento de medición de la calidad; al respecto (Jurán y Gryna, 1999) aseguran que la calidad se debe observar desde la perspectiva del cliente ya que para que un producto se considerado de calidad debe satisfacer las necesidades de los clientes. Por su parte (Militaru y Zanfir, 2016) basados en la ISO 9000 (2015), indican que la calidad es el grado de cumplimiento de los requisitos a partir de un cumulo de características presentes en un servicio o producto.

Vásquez (2007) asegura que la calidad debe ser entendida por los gerentes como la meta cuyo significado e importancia es medible solo a través de la satisfacción de los clientes; dicha satisfacción solo consigue cuando las organizaciones desarrollan procesos eficientes que le permiten ser competitivas en la industria.

Por su parte Barker (1997) asegura que la calidad es la clave para entrar, competir y conseguir el éxito en los mercados actuales y futuros; de acuerdo con esta perspectiva la calidad es la única medio a través cual se puede satisfacer a los consumidores.

La (ISO) en su norma 8402, comprende la calidad como el cumulo de características de las organizaciones que le otorgan la capacidad de cumplir las necesidades implícitas como explicitas.

A través de los conceptos mencionados se puede observar el vínculo existente entre la Cali- 
dad y la estrategia, siendo esta última de acuerdo con (Sarmiento, 2018) una herramienta que fortalece e incrementa el nivel de competitividad y productividad de las empresas; de este modo la calidad como estrategia se encuentra constituida por planes, procesos y actividades cuyo propósito orienta el accionar de las organizaciones hacia el desarrollo de productos o/y servicios óptimos, capaces de satisfacer los requerimientos de los clientes, quienes son en definitiva los que establecen la existencia o no de la variable calidad en un producto.

Luego de abordado el concepto de calidad, se extrapola el mismo al ámbito de la educación Universitaria recorriendo de este modo los referentes más recientes de esta área del conocimiento.

\section{Calidad académica}

Es necesario después de observar los distintos enfoques de calidad, abordar la calidad desde su aplicación en la academia por lo cual se hace necesario citar la Ley Orgánica General del Sistema Educativo (LOGSE) propia de España, la cual establece las variables a tener en cuenta para evaluar la calidad académica. Dichas variables son las siguientes

- Formación docente (habilidades y competencias).

- Los recursos soporte para el desarrollo de las clases y la función directiva.

- Enfoque a la investigación formativa.

- Formación integral.

- Sistema de control.

- Auto evaluación del sistema académico.

\section{Metodología}

\section{Método y enfoque de investigación}

Los diversos enfoques teóricos acerca de la calidad académica, comparados con la calidad del desempeño, nos permitirán observar el nivel de cumplimiento de la Universidad frente al servicio mostrado, lo anterior es conocido como método deductivo. Aunado a lo anterior es pertinente indicar que el enfoque de la investigación es cuantitativo, y se basa en la percepción de los estudiantes, la cual se recopila a través de una encuesta.

\section{Diseño de la investigación}

Como ha quedado indicado en el aparte anterior la investigación se basa en la percepción de los estudiantes de quinto a decimo semestre de la facultad de ingeniería de sistemas.

\section{Población y muestra}

La población objeto de estudio está compuesta por 290 estudiantes del programa de ingeniería de sistemas de segundo a decimo semestre. El marco muestral se calcula a través de la siguiente formula.

$$
\begin{gathered}
n=\frac{N * Z^{2} * p * q}{d^{2} *(N-1)+Z^{2} * p * q} \\
\mathrm{~N}=\text { Población }=290 \\
\mathrm{Z} \alpha=90 \%=1,65 \\
\mathrm{p}=10 \%=0.1 \\
\mathrm{q}=0.9(1-\mathrm{p}) \\
\mathrm{d}=0,03 \\
n=\frac{290 * 1,65^{2} * 0,1 * 0,9}{0,03^{2} *(290-1)+1,65^{2} * 0,1 * 0,9}=140
\end{gathered}
$$

A partir del anterior cálculo se pudo determinar que la muestra para la presente investigación es de 140 estudiantes. 


\section{Recolección y procesamiento datos}

Con miras a la recolección de datos se diseñó una encuesta a partir de la encuesta de satisfacción de estudiantes de programas académicos de posgrado desarrollada por Universidad Javeriana (2014); se seleccionaron cuatro de las variables las cuales fueron evaluada por los estudiantes universitarios, como derivación de esta resultaron 22 unidades de análisis, cada una con su respectiva pregunta; en la tabla $\mathbf{1}$, se pueden observar las variables, las preguntas y las abreviaciones de las unidades de análisis.

Tabla 1. Mapa de Variables

VAR. $\quad$ PREGUNTAS

La Universidad está comprometida con los principios formativos declarados en su misión

La Universidad ejerce la docencia, la investigación y el servicio con excelencia

La Universidad contribuye a la formación integral y no solo especializada

La Universidad contribuye a la creación, desarrollo de la cultura investigativa

Existe compromiso de los directivos con la administración y gestión de la Universidad

Existen los espacios de participación a través de los cuales los estudiantes, docentes, directivos y administrativos tratan los problemas que afectan a la Universidad

Existe una adecuada comunicación hacia toda la comunidad respecto a las decisiones tomadas por la Universidad

La Universidad cuenta con consejería académica

Cuenta la Universidad con horario adecuados para el desarrollo de tutorías

Los planes de estudio son periódicamente actualizados en concordancia con las tendencias que marca el mercado laboral

La universidad cuenta con flexibilidad para cursar asignaturas de los planes de estudio

Es adecuada la metodología de enseñanza empleada en el desarrollo de los planes de estudio

Existen las condiciones para la formación interdisciplinaria.

Es coherente la metodología de evaluación frente a la metodología de enseñanza

Los docentes están cualificados para el desarrollo de procesos de investigación formativa

Los docentes cuentan con las competencias y habilidades para el desarrollo eficiente de los planes de estudio

Existen mecanismos para la evaluación de los profesores por pate del estudiante.

Es adecuada el área de bibliotecas: consigue los textos adecuados para el desarrollo de los planes de estudio

Es adecuada el área de salas de cómputo: cuenta con los equipos, software y ayudas necesarias para el desarrollo de sus actividades académicas

Los salones cuentan con las condiciones necesarias para el desarrollo de las clases (luminosidad, buena ventilación, condiciones de las sillas y un buen tablero)

Son adecuados los auditorios (luminosidad, buena ventilación, condiciones de las sillas)

Cuenta la Universidad con espacios de adecuados para el desarrollo de actividades extracurriculares y de esparcimiento
ABREV.

PRINFORMA

EXCELENCIA

FORMAINTE

CULTUINVES

COMPDIRECT

PARTICIPA

ADECUCOMUN

CONSACADE

HORATUTOR

PLANEESTU

FEXIBCURIC

METOENSE

FORMAINTERDIS

METOEVALU

INVFORMAT

FORMADOCENT

EVALDOCENT

BIBLIOTECA

SALACOMPUTO

SALONES

AUDITORIO

ESPAADECUA

Fuente: elaboración propia, a partir de Universidad Javeriana 2014.

Para el diseño del instrumento se empleó la escala Likert, pues facilito la tabulación y posterior análisis de la información. 


\section{Resultados}

En este punto se muestran los resultados obtenidos a partir de la encuesta realizada a la muestra.

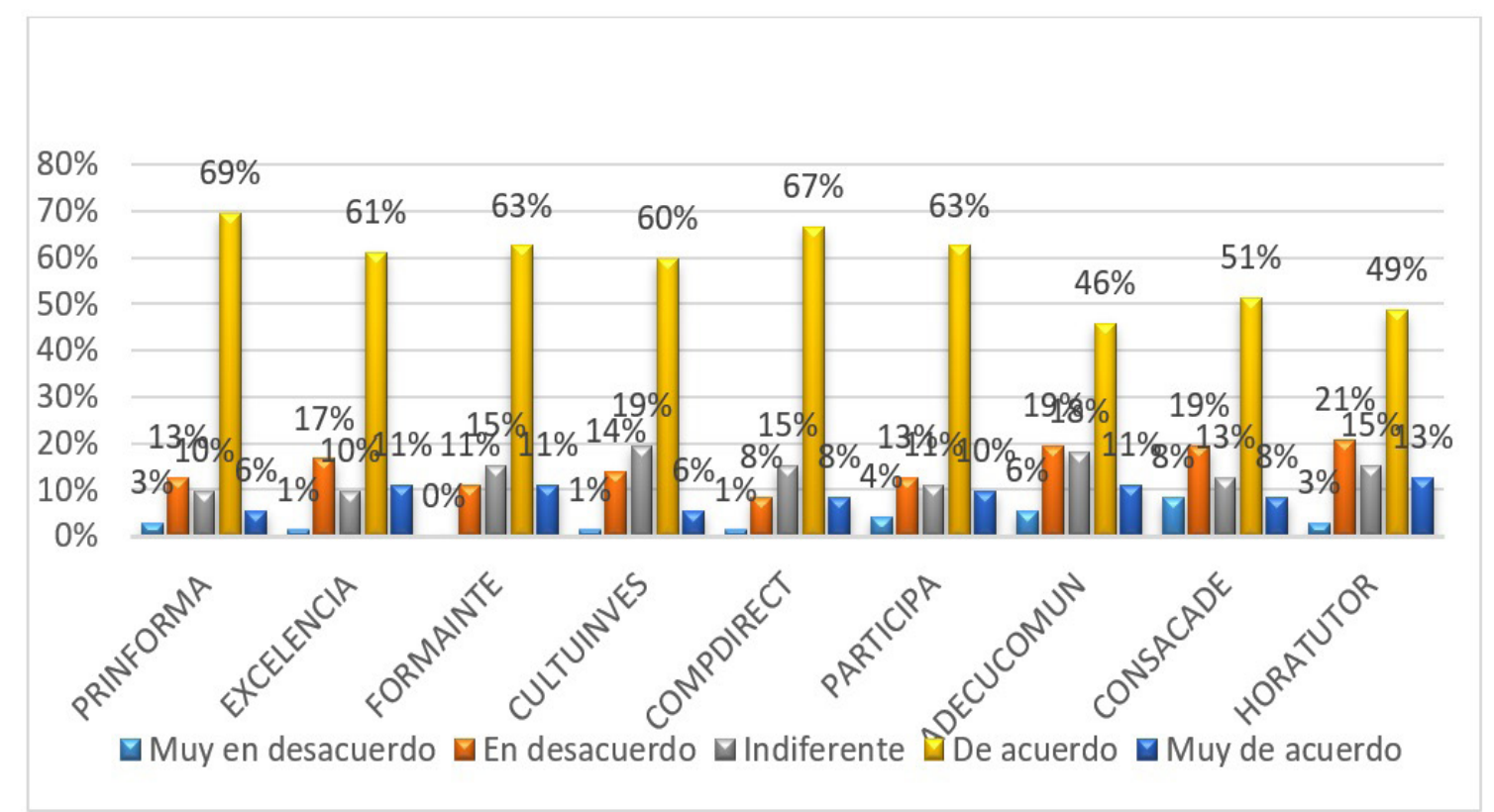

\section{Gráfica 1. Procesos administrativos}

Fuente: elaboración propia 2019

Es claro que la percepción de los estudiantes entorno a la variable proceso administrativos es significativamente positiva, mostrando picos por encima del 45 para la opción de respuesta de acuerdo en las unidades de análisis que com- ponen dicha variable. Por lo anterior se podría afirmar que la Universidad se preocupa por el desarrollo de prácticas de calidad en las distintas actividades administrativas.

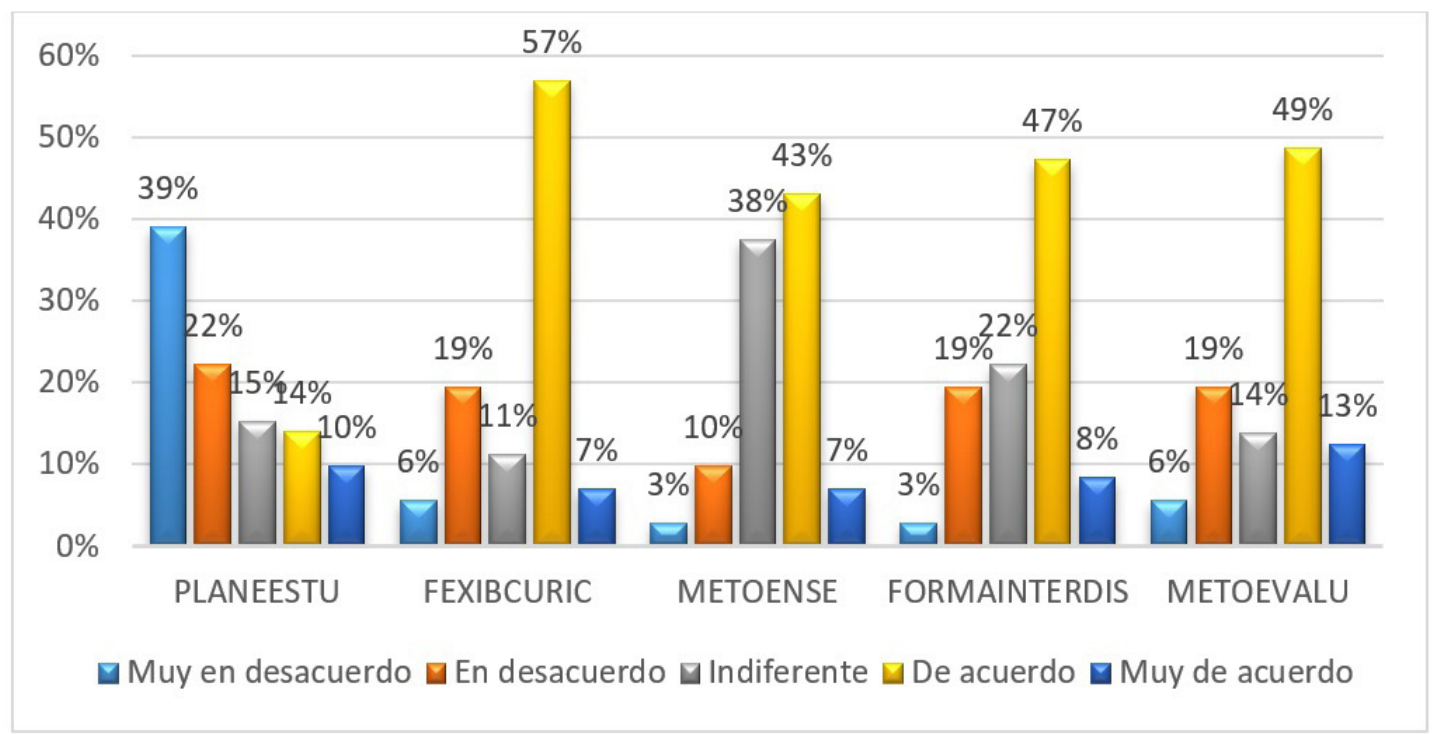

\section{Gráfica 2. Currículo}

Fuente: elaboración propia 2019

Con excepción a la unidad de análisis planes estudios actualizados, la variable currículo muestrea aprobación de los estudiantes. La unidad de análisis planes de estudio con un 39\% para la op- ción "muy en desacuerdo" permite ver la existencia de falencia referente a procesos de naturaleza académica, este es uno punto realmente demanda atención por parte de las autoridades académicas. 


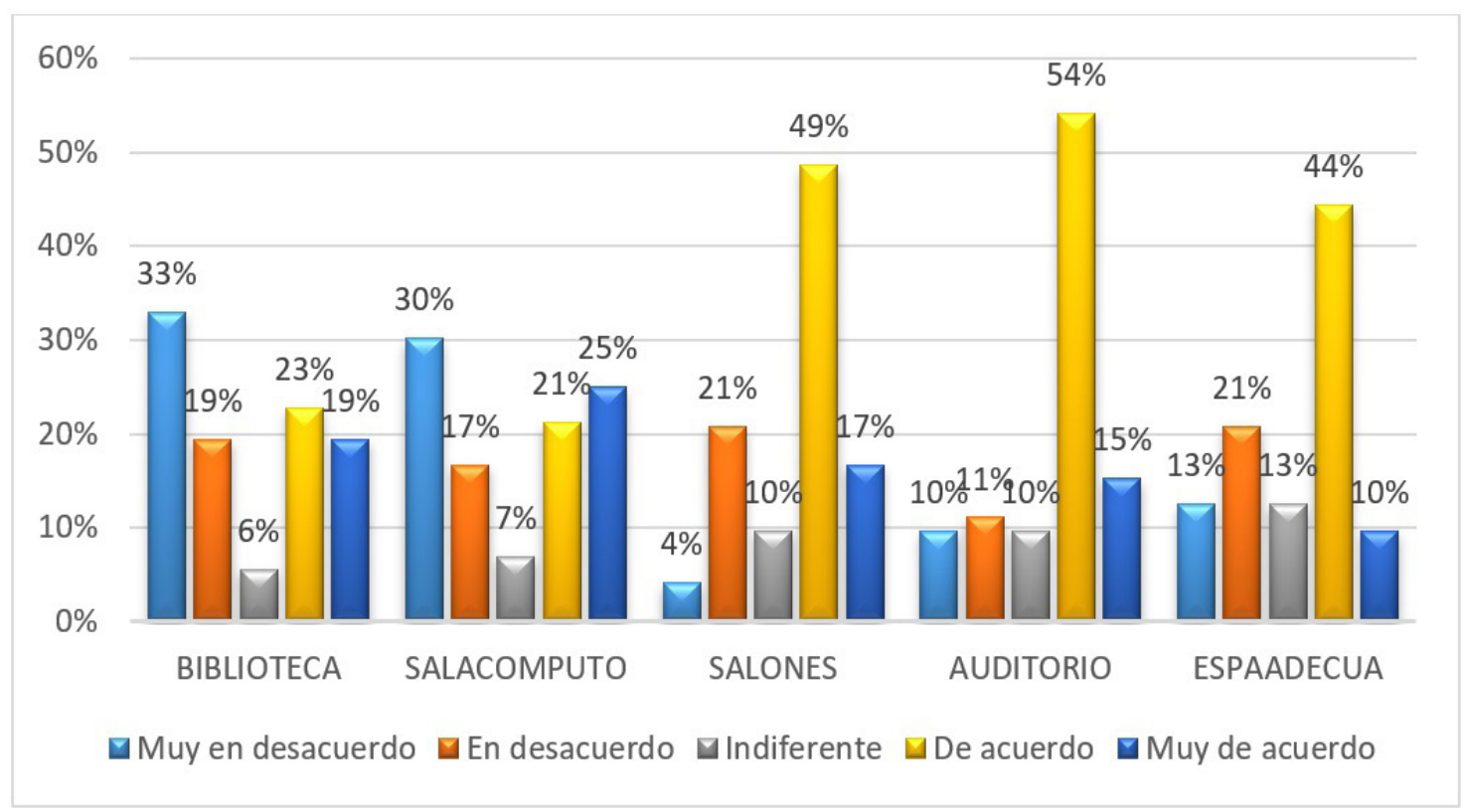

\section{Gráfica 3. Infraestructura}

Fuente: elaboración propia 2019

Falta una mayor inversión por parte de la admi- salones, auditorio, espacios de diversión y esnistración para hacer frente aspectos relaciona- parcimiento son percibidos por los estudiantes dos con la estructura física y equipos de dota- como adecuados para el desarrollo de las activición, los resultados para las unidades de análisis dades académicas y no académicas.

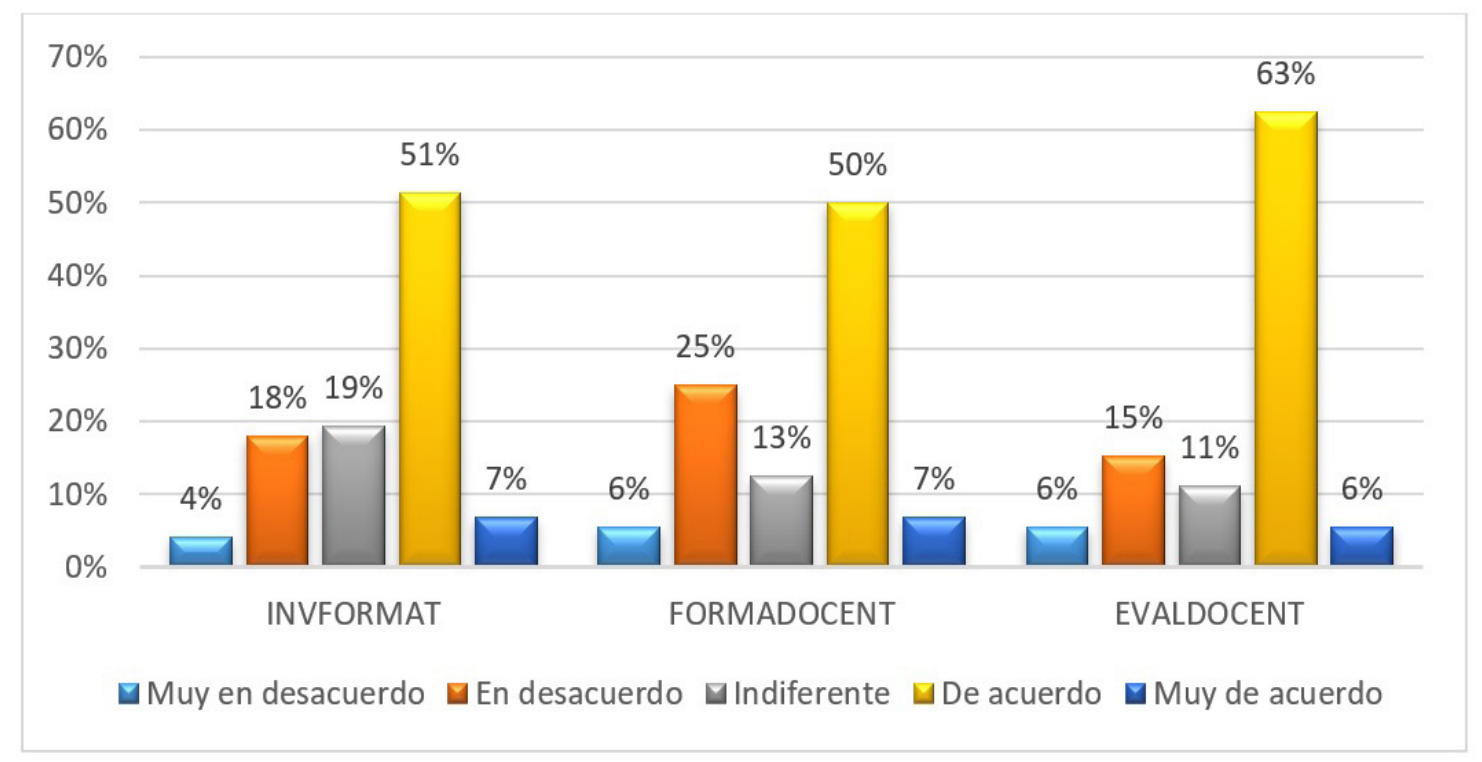

\section{Gráfica 4. Formación docente}

Fuente: elaboración propia 2019

Es interesante observar que la universidad promueve mecanismos por medio de los cuales los alumnos puede evaluar el desempeño de los docentes, esto seguramente facilita la retroalimentación; de otra parte, se puede ob- servar que en la Universidad se preocupa por generar cultura investigativa, ello se ve reforzado puesto que los docentes poseen las competencias y habilidades en investigación. 
René Ramírez Fernández, Jhorquis Machado Licona, Oscar Eduardo Fernández Ramírez

\section{Análisis de correspondencia}

Para un mejor análisis del comportamiento de portamiento de las variables a partir del agrulas variables del estudio se implementa el aná- pamiento de las misma en dimensiones, ello se lisis de correspondencia, al respecto Fernández puede observar a partir de la gráfica de medidas (2011), lo concibe como una herramienta que le discriminantes. (ver grafica 5) (ver abreviafacilita a los investigadores comprender el com- ción en tabla 1)

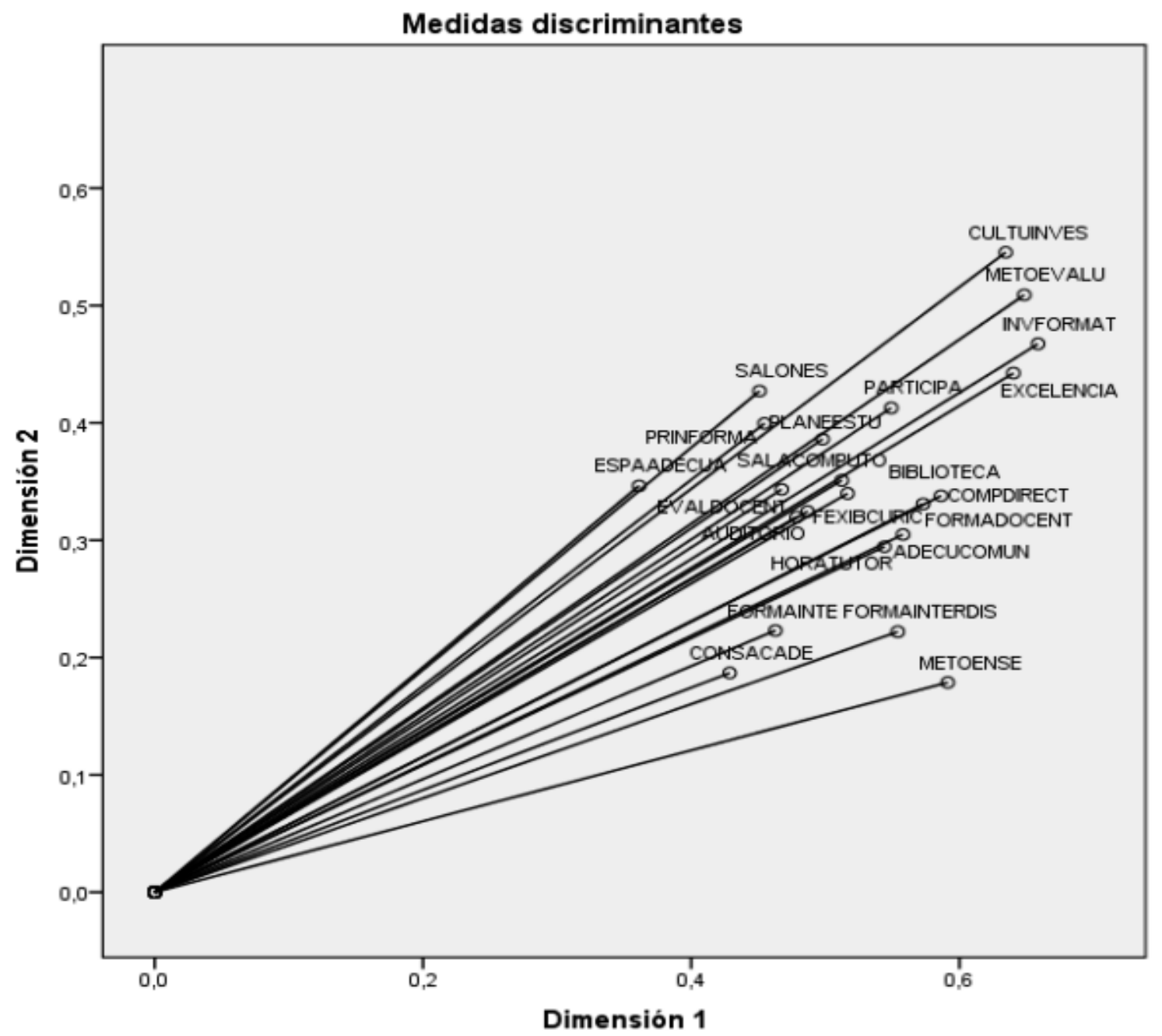

Normalización de principal de variable.

\section{Gráfica 5. Medidas discriminantes}

Fuente: elaboración propia, a través de SPSS (2019)

A partir de lo observado en la gráfica 5 , podemos observar que las unidades de análisis se agrupan en la dimensión uno, lo cual indica que las variables muestran un comportamiento similar; cabe resaltar que las unidades de análisis relacionadas con los procesos investigativos, mostraron una relación más estrecha, aportando significativamente a las dos dimensiones, lo que es consistente con los hallazgos observables las gráficas 1 a 4 . La tabla 2 muestra los coeficientes para las medidas discriminantes, en relación con las unidades de análisis, comprobando así la distribución de estas dentro de las dos dimensiones. 
Tabla2. Medidas discriminantes

\begin{tabular}{llcl}
\hline & \multicolumn{3}{c}{ Dimensión } \\
& $\mathbf{1}$ & $\mathbf{2}$ & Media \\
\hline PRINFORMA & 0,498 & 0,386 & 0,442 \\
EXCELENCIA & 0,640 & 0,442 & 0,541 \\
FORMAINTE & 0,463 & 0,223 & 0,343 \\
CULTUINVES & 0,634 & 0,545 & 0,590 \\
COMPDIRECT & 0,586 & 0,338 & 0,462 \\
PARTICIPA & 0,549 & 0,413 & 0,481 \\
ADECUCOMUN & 0,558 & 0,305 & 0,431 \\
PLANEESTU & 0,455 & 0,400 & 0,427 \\
FEXIBCURIC & 0,487 & 0,325 & 0,406 \\
CONSACADE & 0,429 & 0,187 & 0,308 \\
METOENSE & 0,591 & 0,179 & 0,385 \\
INVFORMAT & 0,659 & 0,467 & 0,563 \\
FORMAINTERDIS & 0,554 & 0,222 & 0,388 \\
FORMADOCENT & 0,573 & 0,331 & 0,452 \\
HORATUTOR & 0,544 & 0,294 & 0,419 \\
METOEVALU & 0,648 & 0,509 & 0,579 \\
EVALDOCENT & 0,468 & 0,343 & 0,405 \\
BIBLIOTECA & 0,517 & 0,340 & 0,428 \\
SALACOMPUTO & 0,512 & 0,351 & 0,432 \\
SALONES & 0,451 & 0,427 & 0,439 \\
AUDITORIO & 0,479 & 0,320 & 0,399 \\
ESPAADECUA & 0,361 & 0,346 & 0,354 \\
Total activo & 11,656 & 7,693 & 9,675 \\
\% de varianza & 52,982 & 34,970 & 43,976 \\
\hline FUente: & & $5 S 5,2019$ &
\end{tabular}

Fuente: elaboración propia, a través del software SPSS, 2019.

\section{Conclusiones}

La universidad debe integrar en sus decisiones administrativas el adoptar un plan que permita mantener actualizados los contenidos impartidos en las aulas de clase, realizando estudios periódicos de las tendencias que dicta el mercado laboral, un ejemplo claro de ello puede consistir en identificar cuáles son los lenguajes de programación más empleados por las empresas para diseñar software a la medida, de mismo lodo identificar cuáles son las herramientas para trabajo en redes de comunicación más empleadas por las empresas; todo ese esfuerzo conlleva a estudiantes con conocimientos ac- tualizados, que puedan hacer frente al mercado laboral. Por otra parte, la Universidad debe destinar los fondos a la modernización de la biblioteca, así como dotarla de libros actuales; la Universidad debe velar por brindar espacios donde en el estudiante pueda socializar con todo el personal de la Universidad. Es evidente la necesidad de cambios en la infraestructura Universitaria.

Finalmente cabe reconocer el esfuerzo de la Universidad en el área investigativa formativa, logrando con ellos promover el hábito investigativo en su comunidad, lo que se ve apoyado en la planta docente. 


\section{Referencias bibliográficas}

Camacaro, P. R. (2010). Hacia la epistemología de la gerencia. Contribuciones a la Economía, (2010$03)$.

De La Lengua, R. A. E. (2010). Diccionario. Vigésima.

Evans, J. R., Lindsay, W. M., Fragoso, F. S., \& Díaz, G. H. (2000). Administración y control de la calidad.

Fernández, R. S. M. Análisis de correspondencias simples y múltiples. Universidad Autónoma de Madrid: Facultad de Ciencias Económicas y Empresariales (2011)

Ishikawa, K. (1988). Qué es el control total de calidad: la modalidad japonesa. Norma.

Juran, J. M., \& Gryna, F. M. (1999). Análisis y planeación de la calidad del desarrollo del producto al uso (No. TS156. J8618 1995.). McGraw-hill.

Militaru, C., \& Zanfir, A. (2016). The vision of new Iso 9000: 2015 standards. Knowledge Horizons. Economics, 8(1), 131.

Sarmiento, L. M. D. (2018). Gestión estratégica de proveedores para fortalecer el proceso de compras en la línea de negocios de reparación y mantenimiento de embarcaciones en COTECMAR. Revista científica anfibios, 1(2), 44-52.

Spain. (1990). Ley orgánica 1/1990, de 3 de octubre, de ordenación general del sistema educativo. Boletín Oficial del Estado.

Universidad Javeriana (2014). Encuesta de satisfacción de estudiantes de programas académicos de posgrado - Pontificia Universidad Javeriana - Sede Central. Consultado el 30 de septiembre del 2019. Disponible en: http://bit.ly/2IPVxxg

Vásquez, M. (2007). La Calidad, el concepto actual que debe ser manejado adecuadamente por los gerentes y funcionarios de toda organización. Consultado el 28 de septiembre del 2019. Disponible en: http://www.degerencia.com/articulo/la_calidad,_el_concepto_actual 